\title{
ESTUDOS DE CULTURA MATERIAL APLICADOS À COLEÇÃO DE TELEGRAFIA DO MUSEU CORREIOS
}

\author{
Bernardo de Barros Arribada* \\ Museu Correios \\ Faculdade de Ciência da Informação da Universidade de Brasília (FCl/UnB) \\ Lillian Maria Araújo de Rezende Alvares \\ Instituto Brasileiro de Informação em Ciência e Tecnologia (IBICT)
}

Programa de Pós-Graduação em Ciência da Informação da Universidade deBrasília (PPGCINF)

\begin{abstract}
RESUMO
Análise de métodos da cultura material para estudo dos objetos museológicos do acervo histórico de telegrafia do Museu Correios, de forma a verificar sua aplicação como ferramenta para a Documentação e Informação em Museus. A metodologia utilizada foi baseada na análise documentária, realizando a comparação entre os modelos a partir de parâmetros pré-estabelecidos. Verificou-se que dos seis modelos estudados, nenhum é totalmente aplicável à coleções de ciência e tecnologia, sendo necessárias adaptações para aplicação no acervo dos museus de ciência e tecnologia, em especial de telecomunicações.
\end{abstract}

\section{PALAVRAS-CHAVE}

Documentação e informação em Museus; Cultura material; Patrimônio de Ciência e Tecnologia;Telegrafia; Museu Correios.

\begin{abstract}
Cultural material methods analysis for the study of the Museu Correios telegraphic historical collections of museological objects, to verify its application as a tool for museum documentation and information. The methodology employed was based on documentary analysis, developing a comparison between the models held from pre-established parameters. It was verified that, from the six models studied, none of them is totally applicable to science and technology collections, with necessary changes to its application in this kind of collections and museums, especially to telecommunication museums.
\end{abstract}

\section{KEY-WORDS}

Museum Documentation and Information. Material Culture. Science and Technology Heritage. Telegraph. Museu Correios.

*bernarribada@uol.com.br; lillian@alvarestech.com. 


\section{Introdução}

A definição de museu do Conselho Internacional de Museus (ICOM) prevê, entre outras coisas, a natureza preservacionista da instituição, à medida que uma de suas obrigações é a salvaguarda de todo testemunho, material e imaterial, do homem e da sociedade. Dentre as atividades previstas para responder a essa premissa, os museus desenvolvem pesquisas e documentam toda e qualquer peça que componha suas coleções.

Segundo Mensch (1994), uma das contribuições da teoria da museologia na atual operacionalização dos museus é o desenvolvimento de metodologias específicas para suas atividades. Instituições normativas, como o Comitê para Documentação (CIDOC/ICOM), desenvolvem guias e padrões específicos para as atividades museológicas.

No entanto, verificam-se, ainda, algumas lacunas no que diz respeito à elaboração de normativas de trabalho, como por exemplo uma metodologia para análise das coleções de ciência e tecnologia, atividade desenvolvida no âmbito da documentação.

O presente artigo tem como objetivo a publicação dos resultados de pesquisa, desenvolvida em âmbito de mestrado em Ciência da Informação, na qual foram analisados seis modelos de estudos de Cultura Material desenvolvidos por profissionais de diferentes campos do conhecimento, como a Antropologia, a Arqueologia e as Artes Aplicadas, buscando identificar um modelo que pudesse ser aplicado no contexto dos museus de ciência e tecnologia, mais especificamente em coleções de telecomunicações, tendo como referencial teórico o valor documental dos objetos do chamado patrimônio de ciência e tecnologia que compõem essa tipologia de acervo.Vale ressaltar, também como motivação para o desenvolvimento do trabalho em tela, a necessidade de identificar melhores práticas para o tratamento da coleção de telegrafia do Museu Correios, único representante oficial dessa parcela da história das telecomunicações nacional.

Para apresentar os resultados da pesquisa, esse artigo foi dividido em duas partes: a primeira buscou compilar as principais ideias que nortearam o trabalho, em especial o valor documental dos objetos de museu, a documentação em museus e os estudos de cultura material, identificando os modelos de estudo do objeto analisados na pesquisa. Cabe ressaltar que os modelos analisados foram selecionados a partir do trabalho da pesquisadora Susan Pearce cuja produção acadêmica gira em torno da aplicabilidade de modelos de Cultura Material em âmbito museológico - especificamente em museus de história. Em sua produção, Pearce identificou seis modelos aplicáveis em âmbito museológico, a saber:J. Prown, R. Batchelor, C. Montgomery, D. Pye, R. Elliot e S. Pearce'. No segundo momento, são apresentados os parâmetros metodológicos estabelecidos na pesquisa e os resultados alcançados, respondendo aos objetivos da pesquisa.

\section{Objetos-documentos? A documentação e informação no museu}

O termo ‘documento' vem do latim docere - ensinar.É possível encontrar em diversas áreas do conhecimento, como o Direito e a Arqueologia, porém com acepções e naturezas específicas em cada área. A museologia se apropria do conceito de documento desenvolvido pela área da Documentação, mais precisamente à definição elaborada por Paul Otlet, autor do livro Traité de Documentation (1934), no qual teoriza e define práticas para o tratamento dos documentos e de seus conteúdos informacionais, prevendo, inclusive, mecanismos para sua recuperação (acesso).

\footnotetext{
Os modelos foram publicados nos seguintes livros de Pearce: Museum, Objects and Collections (1992); Interpreting Objects and Collections (1994).
} 
De maneira geral, para o autor, documento é o "registro do pensamento humano e da realidade exterior em elementos de natureza material" 2 (OTLET, 1934: 10, tradução nossa) - mais precisamente em forma de livros. Ao desenvolver o conceito de documento e suas especificidades, Otlet inclui os objetos museológicos na categoria documentos ditos substitutos dos livros:

a) O documento escrito ou gráfico é a representação das coisas materiais ou das imagens intelectuais e abstratas das coisas. As coisas materiais em si (objetos) podem ser tidas como documentos uma vez que são criadas como elementos sensíveis, resultado de estudos, ou de provas de uma manifestação. É, então, 'documentação objetiva' e 'documentação automática'.

b) Os objetos de todas as espécies dão lugar à coleções. Assim, os objetos naturais: minerais (elementos e compostos químicos, rochas), plantas (ervas, madeiras, raízes), animais (anatomia, teratologia). Assim os objetos criados pelo homem: matéria, produtos, objetos técnicos. [...] (OTLET, 1934: 217, tradução nossa)

E vai além quando indica que nos museus "[...] as coisas reunidas por inteiro, ou amostras (parte de uma matéria homogênea) constituem documentos [...] e que são úteis ao estudo, ao ensino, à pesquisa" (OTLET, 1934: 356, tradução nossa), prevendo o que viria a ser o trinômio principal das atividades museológicas: conservação, pesquisa e comunicação (MENSCH, 1992).

Alguns anos depois, em 195I, a francesa Suzanne Briet, seguidora de Otlet escreve seu manifesto Qu'est-ce que la documentation seguindo a mesma linha de categorização dos objetos enquanto documentos, sendo possível, inclusive, perceber no texto aspectos dos conceitos de objeto museológico e de musealização - objeto re-significado e deslocado de seu ambiente original para um ambiente institucionalizado (Museu) - quando ela defende que:

[...] uma estrela é um documento? Um seixo levado pela corrente é um documento? Um animal vivo é um documento? Não. Mas são documentos as fotografias e os catálogos de estrelas, as pedras de um museu de mineralogia, os animais catalogados e expostos em um zoológico. (BRIET, 195I: 7, tradução nossa)

Os objetos no museu são identificados no Código de Ética para Museus do ICOM (20I3:6) como testemunhos primários, ou seja, objetos-documentos (LIMA, 2008: 37) na medida em que atuam com função de comunicação dos "[...] processos sociais e peculiaridades de caráter expressivos" (LIMA, 2008: 37). Os objetos são portadores de dados, devendo ser tomados, então, como unidades informacionais.

Mensch (1986: 36) afirma que "[...] todo objeto tem um valor, seja intrínseco ou extrínseco, isto é, em conexão com outros objetos", e ainda que "[...] uma lista inicial [desses valores] já demonstra as múltiplas possibilidade: científico, histórico, recreacional, estético, econômico, de valores éticos, etc." (MENSCH, 1986: 36, tradução nossa)

Em uma primeira instância o valor de um objeto é determinado pela suas propriedades materiais... 'Hardware' (informação intrínseca) e 'sofware' [sic] (documentação e informação contextual) juntos geram o 'testemunho' de um objeto. (MENSCH, 1986: 36, tradução nossa)

O que o autor chama de hardware e software são os níveis de informação dos objetos, também conhecidos como informação intrínseca e extrínseca (MENSCH, 1986; FERREZ, I994), do objeto e sobre o objeto (LIMA, 2003), ou ainda dados sintáticos e semânticos. (SOUZA, 2009).

\footnotetext{
${ }^{2} \mathrm{Na}$ ocasião ainda não havia nenhuma discussão acerca da imaterialidade do patrimônio.
} 
Mensch desenvolveu uma abordagem que inclui três níveis de informação, a saber:

Tabela I - Níveis de Informação segundo Peter van Mensch (I 986:4I)

\begin{tabular}{|l|l|}
\hline Nível da Informação & \multicolumn{1}{c|}{ Categorias de Informação } \\
\hline \multirow{4}{*}{ Propriedades Físicas } & Composição, Material \\
\cline { 2 - 2 } & Construção, Técnica \\
\cline { 2 - 2 } & $\begin{array}{l}\text { Morfologia (subdividida em formato e dimensão; estrutura da } \\
\text { superfície; cor/ padrões de cor e imagem; texto) }\end{array}$ \\
\hline \multirow{4}{*}{ Função e Significado } & $\begin{array}{l}\text { Significado primário (significado funcional e significado } \\
\text { expressivo - valor emocional) }\end{array}$ \\
\cline { 2 - 2 } & $\begin{array}{l}\text { Significado secundário (significado simbólico, significado } \\
\text { metafísico) }\end{array}$ \\
\hline \multirow{3}{*}{ História } & $\begin{array}{l}\text { Gênese (o processo no qual a idéia e a matéria-prima são } \\
\text { forjadas à forma) }\end{array}$ \\
\hline \multirow{3}{*}{ História } & $\begin{array}{l}\text { Uso (primeira função - geralmente de acordo com a intenção } \\
\text { do criador; re-utilização) }\end{array}$ \\
\cline { 2 - 2 } & Deterioração (fatores endógenos e exógenos) \\
\cline { 2 - 2 } & Conservação e Restauração \\
\hline
\end{tabular}

Ao serem selecionados para compor uma coleção museológica, aos objetos é agregado, então, um novo valor: o valor documental. Ou seja, eles passam a ser considerados documentos, fontes de informação. No entanto, para que objetos e todo o seu espectro informacional possam ser utilizados na produção de conhecimento, função básica de centros de documentação, é imperativo que passem pelos processos da chamada Documentação em Museus.

As atividades relacionadas à Documentação em Museus se configuram no território da análise das coleções e produção de conhecimento, "[...] que possibilitará a um objeto preservado na asséptica prateleira da reserva técnica ter voz numa exposição e ser efetivamente um elo no processo de transmissão cultural" (GUEDES, 2010: 210). É uma aplicação da teoria da Documentação - e consequentemente da chamada Organização da Informação - à prática museológica. Ou seja, é no âmbito da Documentação que se desenvolvem as atividades conhecidas como Registro, Inventário, Catalogação e Indexação.Assim sendo, as atividades relacionadas à Documentação em Museus se configuram no território da análise da informação (parte da Organização da Informação) dos objetos e das coleções e da produção de conhecimento.

A Documentação em Museus é definida como o "[...] conjunto de informações sobre cada um dos seus itens [dos itens da coleção, dos exemplares/ objetos] e, por conseguinte, a representação destes por meio da palavra e da imagem (fotografia)" (FERREZ, I 994: 65). E, como todas as atividades das instituições museológicas, são regidas por normas de conduta previamente estabelecidas, previstas, inclusive, no código deontológico da área e em documentos da legislação nacional. ${ }^{3}$

De acordo com o CIDOC/ICOM (1995), a Documentação objetiva assegurar a responsabilidade com os objetos, auxiliar na segurança das peças, prover um arquivo histórico sobre os objetos e suporte físico e acesso intelectual aos mesmos.

O presente artigo, conforme anteriormente indicado, se concentra nos processos de pesquisa e análise informacional, visando o desenvolvimento de ferramentas que auxiliem nos processos de representação e recuperação da informação.

${ }^{3}$ Lei $n^{\circ}$ II.904, de 2009 , e Decreto $n^{\circ} 8.124$, de 2013, ambos resultado dos trabalhos empreendidos pelo Instituto Brasileiro de Museus (IBRAM) 


\section{Estudos de Cultura Material para Análise das Coleções}

O processo de análise da informação é atividade primordial para que os objetivos a que se propõe a Documentação em Museus possam ser alcançados dentro das normas e padrões estabelecidos pela área.

No entanto, não há, atualmente, metodologia específica para a Análise da Informação Museológica, principalmente em museus de ciência e tecnologia, foco da pesquisa. Algumas instituições, como Museu de Astronomia e Ciências Afins (MAST), no Rio de Janeiro, já desenvolvem pesquisas buscando estabelecer um modelo para análise informacional de suas coleções. Para isso, recorreram aos estudos em Cultura Material. (LOUREIRO, 2007; 20I2; 20I5; LOUREIRO; LOUREIRO; SILVA, 2008; 2009)

Cultura Material tem, por definição, a "[...] manifestação da cultura por meio da produção material” (PROWN, 1993: I, tradução nossa), ou seja, por meio dos objetos. A premissa principal é a de que esses artefatos

[...] refletem, consciente ou inconscientemente, direta ou indiretamente, as crenças dos indivíduos que os encomendam, os fabricam, os compram ou os usam e, por extensão, as crenças da sociedade à qual esses indivíduos pertencem. (PROWN, 1993: I, tradução nossa)

Assim, podemos afirmar que a Cultura Material estuda as coisas, materiais e imateriais, fabricadas, criadas ou modificadas pelo homem em seus contextos, como "[...] signos e símbolos exteriores das ideias particulares [...]" (JULIEN; ROSSELIN, 2005: 13, tradução nossa), buscando demonstrar seu papel crucial como motores para mudança. Segundo Prown, objetos são resultados de causas e materializam crenças: "[...] quando uma sociedade passa por alguma mudança traumática, essa mudança se manifesta por meio de artefatos" (PROWN, I993: I3, tradução nossa).Assim, são considerados um meio mais eficaz da difusão de uma cultura que suas ideologias.

Moles (1972) afirma que o objeto é vetor de comunicação, isto é, a concretização de um grande número de ações do homem na sociedade (MOLES, 1972), e ainda que os objetos se inscrevem "[...] no plano das mensagens que o meio social envia ao indivíduo ou, reciprocamente, que o Homo faber subministra à sociedade global” (MOLES, I 972: I I).

Nessa perspectiva, os objetos materiais e imateriais devem ser entendidos como sistema de comunicação, isto é, sistema simbólico através do qual os indivíduos emitem todo tipo de informações, como, por exemplo, nível tecnológico e posição social. A interpretação dessas informações foi foco de estudo de profissionais das áreas de aplicação da Cultura Material, em especial da Antropologia, Arqueologia, Etnografia e História, tendo como resultado os Estudos de Cultura Material.

Os Estudos de Cultura Material, também conhecidos como Métodos de Análise de Objetos, são metodologias criadas por profissionais que trabalham a temática, com os quais buscam estudar o ciclo completo dos objetos ou grupos de objetos (incluindo coleções) tendo por objetivo identificar toda a trajetória, da idealização até seu consumo ou uso e descarte ${ }^{4}$, incluindo os valores a eles atribuídos. Segundo Gonçalves os objetos materiais circulam permanentemente pela vida social, e esses estudos acompanham esses diversos contextos:"[...] sejam trocas mercantis, sejam trocas cerimoniais, sejam aqueles espaços institucionais e discursivos tais como coleções, os museus e os chamados patrimônios culturais". (GONÇALVES, 2007: I5).

Dos profissionais que atuam em museus e que se debruçaram sobre os Modelos de Análise de Objetos se destaca a inglesa Susan Pearce. Dentre seus muitos trabalhos, Pearce buscou identificar modelos que fossem aplicáveis ao

\footnotetext{
${ }^{4}$ Nesse caso, inclui na categoria descarte, a alienação do meio econômico via patrimonialização e musealização.
} 
contexto museológico, apresentando ferramentas que contribuíssem para os trabalhos nessas instituições, como curadorias de exposição e gestão e análise de coleções. Como resultado, Pearce publicou três livros (PEARCE, 1989; 1992; 1994) onde buscou indicar modelos de análise de objetos desenvolvidos por profissionais de diversas áreas do conhecimento, como Arqueologia e Belas Artes, conforme tabela abaixo.

\section{Tabela 2 - Modelos de Estudo de Objetos propostos por Susan Pearce para aplicação em contexto museológico}

\begin{tabular}{|c|c|c|}
\hline MODELO & $\begin{array}{l}\text { AUTOR DO } \\
\text { MODELO }\end{array}$ & $\begin{array}{l}\text { PUBLICAÇÃO dE SUSAN QUE } \\
\text { REFERENCIA O MODELO }\end{array}$ \\
\hline Not looking at keetles & R. Batchelor & $\begin{array}{l}\text { Museum, Objects and Collections } \\
\text { (1992); Interpreting Objects and } \\
\text { Collections (1994) }\end{array}$ \\
\hline $\begin{array}{l}\text { Towards a material history } \\
\text { methodology }\end{array}$ & R. Elliot & $\begin{array}{l}\text { Museum, Objects and Collections } \\
\text { (1992); Interpreting Objects and } \\
\text { Collections (1994) }\end{array}$ \\
\hline $\begin{array}{l}\text { Artifact study: a proposed } \\
\text { model }\end{array}$ & E. M. Fleming & $\begin{array}{l}\text { Museum, Objects and Collections } \\
\text { (1992) }\end{array}$ \\
\hline $\begin{array}{l}\text { The connoisseurship of } \\
\text { artifacts }\end{array}$ & C. Montgomery & $\begin{array}{l}\text { Museum, Objects and Collections } \\
(1992)\end{array}$ \\
\hline Thinking about things & S. Pearce & $\begin{array}{l}\text { Museum, Objects and Collections } \\
\text { (1992); Interpreting Objects and } \\
\text { Collections (1994) }\end{array}$ \\
\hline $\begin{array}{l}\text { Mind in matters: an } \\
\text { introduction to material } \\
\text { culture theory and method }\end{array}$ & J. Prown & $\begin{array}{l}\text { Museum, Objects and Collections } \\
\text { (1992); Interpreting Objects and } \\
\text { Collections (1994) }\end{array}$ \\
\hline
\end{tabular}

Para a pesquisa em tela, buscou-se analisar esses seis Estudos de Cultura Material levantados por Susan Pearce, e identificar a aplicabilidade em uma tipologia de instituição museológica: Museus de Ciência e Tecnologia, mais especificamente, Museu que possuem acervo de Telecomunicação. $O$ que motivou essa pesquisa é a falta de metodologia específica para análise de objetos de ciência e tecnologia (GRANATO et. al., 2007) e, ao mesmo tempo, uma necessidade urgente que o campo tem de trabalhar melhor essa tipologia de acervo, uma vez que esses museus detém toda a história do desenvolvimento tecnológico de nossa sociedade.

\section{Aspectos teóricos e práticos da metodologia da pesquisa}

A pesquisa aqui apresentada teve como propósito ser um estudo descritivo à medida em que buscou verificar e descrever características de um dado fenômeno - Documentação em Museus, Documentação de Acervo de Ciência e Tecnologia e Documentação no Museu Correios.

A abordagem que se pretendeu tomar para o desenvolvimento do estudo é a abordagem qualitativa, pois o estudo se configurou como um meio para explorar e entender o significado que os indivíduos ou os grupos atribuem a um problema social ou humano, específica e profundamente. Creswell (2003: 26) indica que as pesquisas qualitativas são processos que envolvem os procedimentos emergentes, os dados coletados no ambiente da pesquisa e a análise dos dados indutivamente e as interpretações feitas pelo pesquisador acerca do significado dos dados.

Além de seu propósito descritivo e de sua abordagem qualitativa, a pesquisa aqui desenvolvida apresenta natureza aplicada, tendo como finalidade ge- 
rar produtos - método de análise e representação da informação de coleções telegráficas - e melhorar processos - documentação em museus. Ou seja, busca gerar conhecimentos para aplicação prática e imediata, focando na solução dos problemas que originaram o estudo. Para corroborar com essa afirmativa, Sekaran afirma que as pesquisas aplicadas são pesquisa "[...] com intenção de aplicação dos resultados para solucionar problemas específicos recorrentemente vivenciados em uma organização" (SEKARAN, 2003: 8, tradução nossa).

$\mathrm{Na}$ análise dos métodos de análise do objeto foi utilizada a pesquisa documentária. Essa técnica, que de acordo com Ahmed é muitas vezes ignorada pelos pesquisadores, se refere à análise de documentos que "[...] contém informações sobre o fenômeno que se deseja estudar". (AHMED, 2010 : 2, tradução nossa) ${ }^{5}$

Ahmed defende que esse método de pesquisa é usado para investigar e categorizar fontes físicas, como documentos registrados - escritos. No que diz respeito a esses documentos o autor defende que - "[...] documentos não se mantém sozinhos" (AHMED, 2010: 2, tradução nossa) ${ }^{6}$ - ou seja, para serem usados como fontes de dados para pesquisas, os documentos devem ser situados em modelos de referências teóricas.

A técnica de coleta dos dados incluiu, além da revisão sistemática de literatura já mencionada anteriormente, o levantamento e análise descritiva dos métodos de cultura material, a partir de parâmetros pré-estabelecidos, a saber:

Tabela 3 - Parâmetros para Coleta e Análise dos Dados

\begin{tabular}{|l|l|}
\hline \multicolumn{1}{|c|}{ PARÂMETRo } & \multicolumn{1}{c|}{ DADos Coletados } \\
\hline Origem disciplinar & $\begin{array}{l}\text { Qual a área do conhecimento em que o autor do modelo tra- } \\
\text { balhava ou em qual tipo de objeto pretendia aplicar o estudo. }\end{array}$ \\
\hline Contexto & $\begin{array}{l}\text { O modelo foi desenvolvido em que contexto: se acadêmico, } \\
\text { se em contexto museológico, em seminários ou congressos, } \\
\text { se esse contexto influenciou no desenvolvimento do mo- } \\
\text { delo, ou outro aspecto do ambiente de criação do método. }\end{array}$ \\
\hline $\begin{array}{l}\text { Aplicação em âmbito } \\
\text { museológico }\end{array}$ & $\begin{array}{l}\text { Buscou-se verificar se em algum momento do texto o autor } \\
\text { cita a aplicação em contexto museológico. }\end{array}$ \\
\hline $\begin{array}{l}\text { Metodologia } \\
\text { (aspectos teóricos) }\end{array}$ & $\begin{array}{l}\text { Esse parâmetro é de análise da teoria a ser aplicada no estu- } \\
\text { do do objeto. Como é construído o modelo e se o estudo } \\
\text { proposto está pautado na observação (questão da subjeti- } \\
\text { vidade*) ou da análise de dados objetivos. Inclui, também, } \\
\text { previsão de atuação de outros profissionais e uso de outros } \\
\text { documentos (como, por exemplo, arquivísticos). }\end{array}$ \\
\hline $\begin{array}{l}\text { Metodologia } \\
\text { (aspectos práticos) }\end{array}$ & $\begin{array}{l}\text { Diferentemente do aspecto teórico, aqui se analisa como se } \\
\text { dá o estudo na prática. Quais as açães previstas para e com } \\
\text { o objeto. É análise do próprio desenho da pesquisa prevista } \\
\text { no Método. }\end{array}$ \\
\hline $\begin{array}{l}\text { Níveis de informa- } \\
\text { ção analisados }\end{array}$ & $\begin{array}{l}\text { Tendo como parâmetro para comparação os conceitos de in- } \\
\text { formaçães intrínsecas e informaçães extrínsecas desenvolvi- } \\
\text { dos por Peter van Mensch, no âmbito da Documentação em } \\
\text { Museus, foco de aplicação do modelos de estudo de objeto. }\end{array}$ \\
\hline Produtos & $\begin{array}{l}\text { Verificou-se se o autor propôs a criação de algum do- } \\
\text { cumento, dossiê, ou até mesmo formulário catalográ- } \\
\text { ficos. }\end{array}$ \\
\hline
\end{tabular}

\footnotetext{
5 [...] contains information about phenomenon we wish to study.

6 [...] documents do not stand alone.

*A questão da subjetividade na presente dissertação se justifica na necessidade de informações certificadas no que tange à Documentação dos objetos musealizados, para posterior comunicação e disseminação.
} 


\section{Resultados da pesquisa}

A análise dos seis modelos de estudo do objeto desenvolvida por essa pesquisa teve como principal objetivo identificar qual dessas metodologias é a mais adequada para o tratamento informacional de itens do patrimônio de ciência e tecnologia musealizado. De forma prática, a identificação de um modelo para essa tipologia de coleção responderia ao objetivo específico de apontar ferramenta para a gestão da coleção telegráfica do Museu Correios - em especial nas atividades da Documentação.

O primeiro modelo analisado, o de Charles Montgomery (1999), foi desenvolvido a partir das Artes Plásticas, e se estabeleceu como o resultado de exercícios realizados por alunos universitários que se debruçaram sobre a análise de objetos de Artes Plásticas e Aplicadas. O modelo não apresentou metodologia passível de aplicação em coleções científicas por propor o estudo do objeto a partir de quatorze pontos de análise, por vezes exclusivos do campo de origem do modelo.

Os modelos de Fleming (1974) e de Prown (1982), apesar de terem sido desenvolvidos em contexto museológico - ambos em museus artísticos - se apresentaram passíveis de serem aplicados em museus de ciência e tecnologia, principalmente pelo fato de, mesmo tendo como foco os aspectos estéticos e formais do objeto, orientar o estudo para questões de manufatura, utilização e atribuição de valores. No entanto, as metodologias desenvolvidas pelos dois autores ainda apresentam um grau de subjetividade que impossibilita sua aplicação no âmbito da Gestão das Coleções, em especial da Documentação em Museus, foco desse trabalho. ${ }^{7}$

Batchelor (1994), apesar de ter desenvolvido o seu modelo a partir da demanda de um museu de tecnologia, de apresentar uma proposta ligada ao desenvolvimento do design e da técnica, e de trazer inovações ao estudo do objeto - como a análise das questões mercadológicas - propõe um estudo a partir do levantamento de informações pela observação. Assim, os dados levantados pelo autor se apresentam subjetivos, principalmente pela falta de um fase de certificação profissional das informações coletadas.

Outro aspecto que impossibilita a aplicação do modelo de Batchelor (1994) é o foco do modelo nas informações extrínsecas. Mesmo que o autor indique a identificação das informações intrínsecas, no modelo elas são utilizadas para interpretar as informações extrínsecas, e não como dado relevante da pesquisa.

Já os modelos desenvolvidos por R. Elliot (1985) e Pearce (1994) apresentaram aplicabilidade em museus de ciência e tecnologia, porém com alguns pontos em comum e outros divergentes.

Apesar de se originarem da mesma fonte - o modelo de Fleming (1974) - as metodologias e os esquemas propostos pelos autores são diferentes, no entanto complementares. Ambos apresentam terminologia mais estruturada que a do modelo-base, possibilitando trabalho com menor grau de ambiguidade e ruído na informação

O grupo de R. Elliot (1985), no entanto, se sobressai ao propor a certificação das informações por participação de profissionais das áreas referentes às coleções e apresentar esquema que possibilite um estudo mais dinâmico, uma vez que para chegar ao modelo final, foram realizadas diversas aplicações a fim de verificar erros e inconsistências no modelo. Outro ponto importante

\footnotetext{
${ }^{7}$ A questão da subjetividade em Documentação torna-se cabal a partir do momento que essa atividade oficializa o valor documental do objeto.
} 
da metodologia proposto por R. Elliot é a utilização de gramáticas e terminologias específicas, o que dialoga de forma direta com as questões da linguagem documentária e de linguagem especialidade necessárias às práticas documentais.

Sendo assim, a pesquisa resultou na fusão desses dois modelos, propondo uma nova metodologia passível de aplicação em museu de ciência e tecnologia: o modelo de R. Elliot ( 1985) foi tomado como base para o desenvolvimento do esquema metodológico proposto; no entanto, na composição final, é possível perceber a interação com as fases de estudo do objeto propostas no modelo de Pearce (1994).

Os passos e ações propostos na metodologia desenvolvida na pesquisa possibilitam a coleta dos dados para preenchimento de fichas de informação do objeto presentes em museus de ciência e tecnologia, em especial as fichas elaboradas a partir dos documentos desenvolvido pelo CIDOC, órgão máximo regulador da atividade documental em contexto museológico. É possível identificar, também, que a metodologia aqui proposta contempla os dois níveis de informação definidas por Mensch (1986), e já discutida no presente artigo.

Uma vez que as informações coletadas nos processos de Documentação de coleções musealizadas devem ser as mais completas e certificadas, a metodologia proposta pela pesquisa prevê a utilização de dados de fontes secundárias de informação - ou seja, outros tipos de documentos como bibliográficos e arquivísticos - referência cruzada - e a participação de profissionais nas áreas que respondem pelo acervo - no caso da coleção telegráfica, a participação de engenheiros elétricos ou outros técnicos em eletricidade e em comunicação.

Cabe ressaltar que esse modelo de estudo de objeto se configura como ferramenta para as atividades de gerenciamento de coleções, extrapolando os limites da Documentação em Museus, se fazendo importante para as outras atividades, como a curadoria em exposições.

\section{Considerações finais}

A Documentação em Museus, é conjunto de atividades essenciais aos museus, à medida que atuam em pontos estratégicos para o bom funcionamento das instituições.

Os documentos originados das atividades de registro, catalogação, inventário e indexação atuam no âmbito da administração das coleções, na segurança e, principalmente, no aspecto comunicacional do Museu, como fonte para pesquisas e para o desenvolvimento de ações de comunicação - em especial exposições.

A pesquisa "Tratamento Informacional da Coleção Telegráfica do Museu Correios: análise sob a perspectiva científica, tecnológica e de cultura material”, desenvolvida no âmbito do Programa de Pós-Graduação em Ciência da Informação da Universidade de Brasília, teve como objetivo geral identificar qual modelo de cultura material, desenvolvidos por profissionais que se debruçaram sobre as questões da Cultura Material e referendados para uso em instituições museológicas, é o mais adequado para o tratamento informacional da coleção telegráfica do Museu Correios.

Em contrapartida, esse objetivo buscou fortalecer a interdisciplinaridade entre a Museologia e a Ciência da Informação no que tange ao tratamento informacional dos documentos - objetos de museu -, bem como identificou subsídios para o gerenciamento de coleções de ciência e tecnologia - Patrimônio Científico Musealizado. 
Tendo como foco o tratamento informacional, em especial a análise da informação, dos objetos que compõem a coleção telegráfica, a pesquisa identificou metodologias específicas para estudo de objetos - os Estudo de Cultura Material - elaboradas por profissionais de diversas áreas, como a Antropologia, a Arte e a História, e cuja aplicação em contexto museológico foi corroborada por Susan Pearce, professora do curso de museus da Leicester University.

O objetivo da pesquisa girar em torno da identificação da aplicabilidade desses modelos em museus de ciência e tecnologia se justifica na afirmativa dos pesquisadores de não existência de normativas específicas para as atividades de gestão de suas coleções, em especial da informação museológica.

Dos seis modelos analisados, verificou-se que todos os modelos apresentavam pontos positivos e negativos no que tange sua aplicação em museu de ciência e tecnologia, havendo a necessidade, então, de adequação desses modelos ao ambiente da pesquisa.

Assim, elaborou-se um modelo visando aplicação nessa tipologia de acervo. Uma vez que o Museu Correios apresenta uma coleção composta por exemplares de ciência e tecnologia, a pesquisa desenvolveu, então, ferramenta específica para o tratamento informacional dessa parcela do acervo da instituição.

No entanto, a pesquisa não se pretende definitiva, abrindo caminho para novas possibilidades de estudo e, principalmente, verificação de aplicação desse modelo para qualquer tipologia de acervo, possibilitando, assim, a delimitação de uma ferramenta para análise da informação museológica, independente da tipologia das coleções que compõem o Patrimônio Cultural musealizado.

\section{Referências}

AHMED, J. U. Documentary research methods: new dimensions. Indus Journal of Management \& Social Sciences. 4(I). 20I0. p.I-I4.

BATCHELOR, R. Not looking at keetles. In: PEARCE, S. Interpreting Objects and Collections. London: Routledge, 1994. p.I39-I43.

BRASIL. Lei I I.904, de 14 de janeiro de 2009. Disponível em: <http://www.planalto.gov.br/ccivil_03/_Ato2007-2010/2009/Lei/LI 1904.htm>. Acesso em: I2 jan. 2015.

. Decreto 8.I 24, de I 3 de outubro de 2013. Disponível em: <http://www. planalto.gov.br/ccivil_03/_Ato20II-20I4/20I3/Decreto/D8I24.htm>. Acesso em: I 2 jan. 2015.

BRIET, S. Qu'est-ce que la documentation? Paris: EDIT - Éditions Documentaires, Industrielles et Techniques. 1951.

CRESWELL, J.W. Research Design - Qualitative, quantitative and mixed methods approaches. 2. ed. USA: University of Nebraska, Lincoln. 2003.

INTERNATIONAL COMMITTEE FOR DOCUMENTATION - CIDOC. International Guidelines for museum object information: the CIDOC information categories. 1995. Disponível em: <http://www.cidoc-crm.org/docs/guide.htm>. Acesso m: 12 jan. 2015

ELLIOTT, R. Towards a Material History Methodology. Material Culture Review Revue de la culture matérielle. [S.I.], jun. 1985.

FERREZ, H. D. Documentação Museológica: Teoria para uma boa prática. In: IPHAN. Estudos Museológicos. IPHAN. Rio de Janeiro. 1994. p.65-74. (Caderno de Ensaios 2) 
FLEMING, E. M. F. Artifact Study - A proposed model. Winterthur Portfolio. $\mathrm{n}^{\circ} 9$. 1974. p. I53-I73.

GONÇALVES, J. R. S.. Teorias antropológicas e objetos materiais. In:

Antropologia dos Objetos: coleções, museus e patrimônios. Rio de Janeiro: IPHAN - Instituto do Patrimônio Histórico e Artístico Nacional. 2007. p. I 3-42. (Coleção Museu, Memória e Cidadania)

GRANATO, M. SANTOS, C. P. dos. FURTADO, J. L. GOMES, L. P. Objetos de Ciência e Tecnologia como Fontes Documentais para a História das Ciência: resultados parciais. In: VIII ENANCIB - Encontro Nacional de Pesquisa em Ciência da Informação. Salvador. 28 a 31 de outubro de 2007. Trabalhos Apresentados. Salvador. 2007. n/p. Disponível em: <http://www.enancib.ppgci.ufba.br/artigos/ DMP--035.pdf>. Acesso em: 22 jan. 15.

GUEDES,A. C. Museus, documentação e transmissão cultural. In: MAGALHAES, A. M. BEZERRA, R. Z. BENCHETRIT, S. F. (Orgs.) Museus e Comunicação: Exposição como objeto de estudo. Rio de Janeiro: Museu Histórico Nacional. 2010. p.207-2I 2 .

INTERNATIONAL Council of Museums (ICOM). Code of Ethics for Museums. Buenos Aires, 1986. Barcelona, 200I. Seul, 2004. 2009. Disponível em: http:// icom.museum/the-vision/code-of-ethics/translations/. Acesso em 16 de setembro de 2014.

JULIEN, M-P. ROSSELIN, C. La culture matérielle. Paris: La Découvert. 2005.

LE GOFF, J. Memória e história. $3^{\text {a }}$ Ed. Campinas: UNICAMP. 1994.

LIMA, D. F. C. Ciência da informação, museologia e fertilização interdisciplinar: Informação em Arte, um novo campo do saber. 2003. Tese (Doutorado em Ciência da Informação). IBICT/PPGCI-UFRJ/ECO. Rio de Janeiro. Orientadora: Lena Vania Ribeiro Pinheiro. 358fs.

. Herança cultural (re)interpretada ou a memória social e a instituição museu: releitura e reflexões. Museologia e Patrimônio - Revista Eletrônica do Programa de Pós-Graduação em Museologia e Patrimônio, PPG-PMUS UNIRIO/MAST. Rio de Janeiro, v. I, n. I, 2008. p. 33-43.

LOUREIRO, M. L. N. M. Fragmentos, modelos, imagens: processos de musealização no domínio da ciência. DataGramaZero - Revista de Ciência da Informação. v.8 n. 2. abr. 2007.

. Notas sobre a construção do objeto musealizado como documento. Anais do Museu Histórico Nacional. vol. 44. 2012. p. 91 - 106.

. Musealização e Cultura Material de Ciência \& Tecnologia. Museologia e Patrimônio - Revista Eletrônica do Programa de Pós-Graduação em Museologia e Patrimônio. Unirio. MAST. v.8. n. 2. 2015.

LOUREIRO, M. L. N. M.; LOUREIRO, J. M. M.; SILVA, S. D. Museus, informação e cultura material: o desafio da interdisciplinaridade. In: IX Encontro Nacional de Pesquisa em Ciência da Informação (ENANCIB). São Paulo. Trabalhos Apresentados. São Paulo: USP-Universidade de São Paulo. 2008.

. Apontamentos sobre objetos técnicos como documentos. In: ENANCIB - Encontro Nacional de Ciência da Informação - Responsabilidade social da Ciência da Informação, I0, 2009. Anais eletrônicos... João Pessoa: Ideia/Editora Universitária/UFPB, 2009.

MENSCH, P. van. Museology and the object as a data carrier. Informatologia Yoguslavica. I8 (I-2); I986. p. 35-43. 
. Modelos conceituais de museus e sua relação com o patrimônio natural e cultural. In: ICOFOM-LAM - International Council for Museology - Latin America and Caribbean. Boletim. Buenos Aires. N 4/5. p. IX-X. Ago. 1992. Trad. Tereza Scheiner.

MOLES, A. Objeto e comunicação. In: MOLES, A. et. Al. Semiologia dos objetos. Petrópolis: Ed.Vozes. 1972. p. 9-4I. (Coleção Novas Perspectivas em Comunicação 4)

MONTGOMERY, Charles. The connoisseurship of artifacts. In: SCHLERETH, T. J. Material Culture Studies in America. Maryland:Altamira Pressa. 1999. p. I43-I 52.

OTLET, P. Traité de documentation: le livre sur le livre, théorie et pratique. Bruxelles: Editions Mundaneum. 1934.

PEARCE, S. Museum Studies in Material Culture. London: Leicester University Press, 1989.

. Museums, Objects and Collections: a cultural study. Washington D.C.: Smithsonian Institution Press. 1992. . Interpreting Objects and Collections. London: Routledge, 1994.

PROWN, J. D. Mind in matter: an introduction to material culture theory and method.Winterthur Portfolio. vol. I7. n. I. Spring. 1982. p. I- 19.

.The truth of material culture: history or fiction. In: LUBAR, S. KINGERY, W.D. History from things: essays on material culture. Londres, Washington: Smithsonian Institution Press. 1993. p. I- 19.

SCHLERETH, T. J. Material Culture Studies in America. Maryland: Altamira Pressa. 1999.

SEKARAN, U. Research methods for business: a skill-building approach. Forth Edition. USA: John Wiley \& Sons, Inc. 2003.

SOUZA, D. M.V. Informação e construção de conhecimento no horizonte museológico. DataGramaZero. v. I0. n.6. dez. 2009. 